\title{
An Efficient Approach for Data Gathering and Sharing with Inter Node Communication in Mobile-Sink
}

\author{
Mr. Ravindra K. Gupta ${ }^{1}$, Mr. Shashank Saxena ${ }^{2}$, Dr. Shailendra Singh ${ }^{3}$, \\ Mr. Gajendra Singh ${ }^{4}$, Mr. Ashutosh k. Dubey ${ }^{5}$ \\ ravindra_p84@rediffmail. $\mathrm{com}^{1}$ \\ M.Tech Scholar, CSE, SSSIST, Sehore ${ }^{2}$ \\ shashank.dollar@gmail.com \\ Professor (CSE), NITTTR, Bhopal ${ }^{3}$ \\ ssinghanitttrbpl.ac.in \\ HOD(CSE/IT),Sssist,Sehore ${ }^{4}$ \\ AP, CSE, TITR, Bhopal, India ${ }^{5}$ \\ a shutoshdubey123@gmail. com
}

\begin{abstract}
In today's era Wireless sensor networks (WSNs) have emerged as a solution for a wide range of applications. Most of the traditional WSN architectures consist of static nodes which are densely deployed over a sensing area. Recently, several WSN architectures based on mobile elements (MEs) have been proposed. Most of them exploit mobility to address the problem of data collection in WSNs. The common drawback among them is to data sharing between interconnected nodes. In this paper we propose an Efficient Approach for Data Gathering and Sharing with Inter Node Communication in Mobile-Sink. Our algorithm is divided into seven parts: Registration Phase, Authentication Phase, Request and Reply Phase, Setup Phase, Setup Phase (NN), Data Gathering, and Forwarding to Sink. Our approach provides an efficient way to handle data in between the intercommunication nodes. By the above approach we can access the data from the node which is not in the list, by sharing the data from the node which is approachable to the desired node. For accessing and sharing we need some security so that the data can be shared between authenticated nodes. For this we use two way security approach one for the accessing node and other for the sharing.
\end{abstract}

\section{KEYWORDS}

Sensor Network; Mobile Sink, WSN, Data Gathering

\section{INTRODUCTION}

The WSN is built of nodes from a few to several hundreds or even thousands, where each node is connected to one or sometimes several sensors. Each such sensor network node has typically several parts: a radio transceiver with an internal antenna or connection to an external antenna, a microcontroller, an electronic circuit for interfacing with the sensors and an energy source, usually a battery or an embedded form of energy harvesting. A sensor node might vary in size from that of a shoebox down to the size of a grain of dust, although functioning "motes" of genuine microscopic dimensions have yet to be created. The cost of sensor nodes is similarly variable, ranging from a few to hundreds of dollars, depending on the complexity of the individual sensor nodes. Size and cost constraints on sensor nodes result in corresponding David C. Wyld, et al. (Eds): CCSEA, SEA, CLOUD, DKMP, CS \& IT 05, pp. 331-342, 2012. 
constraints on resources such as energy, memory, computational speed and communications bandwidth.

These sensor devices are resource-constrained, in particular, powered by battery thus energy is the most precious resource of a sensor network since periodically replacing or recharging the battery of the nodes in large scale deployments is infeasible. Therefore, reducing power consumption of sensor nodes then prolonging the useful network lifetime has been a hot topic of extensive study [1]. Past work has focused on developing energy efficient protocols for MAC[1], [2] and routing [3], [4], [5]. Most of network modes considered in their research have a single static base station located on the periphery of the sensor network. Though all these protocols achieve their optimization goals under certain conditions, they are focused on static sensor nodes. We'll show that further improvements on the network lifetime can be achieved using mobility.

Sensor networks are wireless networks consisting of a large number of miniature electromechanical devices (sensors) with sensing, computing and communication capabilities [6, 7]. They can be used for collecting sensory information, such as temperature measurements, from an extended geographic area.

Operating a sensor network poses algorithmic challenges due to the limited capabilities of the sensor devices. In data gathering problems the focus is in maximizing the amount of data gathered from the sensors to a base station using direct or multi-hop radio transmission, under one or more limiting factors.

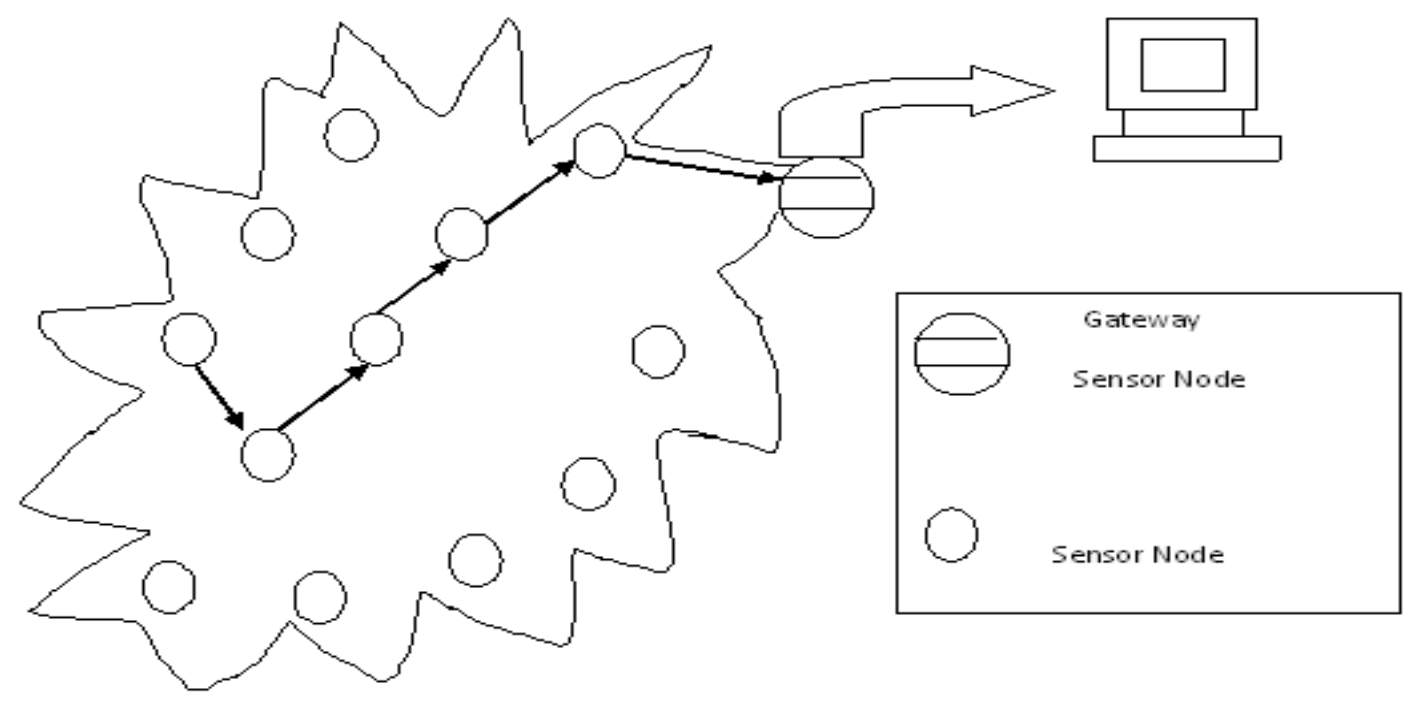

Figure 1: Wireless Sensor Network

In this paper we provide an overview of several aspects about previous research. The rest of this paper is arranged as follows: Section 2 introduces Data Gathering in WSN; Section 3 describes about Literature Survey; Section 4 shows Mobile sink with java Environment; Section 5 describes about proposed method. Section 6 describes Conclusion and Future prospect. 


\section{DATA GATHERING IN WSN}

The process of grouping the sensor nodes in a densely deployed large-scale sensor network is known as clustering. The intelligent way to combine and compress the data belonging to a single cluster is known as data aggregation in cluster based environment. There are some issues involved with the process of clustering in a wireless sensor network. First issue is, how many clusters should be formed that could optimize some performance parameter. Second could be how many nodes should be taken in to a single cluster. Third important issue is the selection procedure of cluster-head in a cluster. Another issue is that user can put some more powerful nodes, in terms of energy, in the network which can act as a cluster-head and other simple node work as clustermember only.

In Data Aggregation, value is derived from the aggregation of two or more contributing data characteristics.

Aggregation can be made from different data occurrences within the same data subject, business transactions and a denormalized database and between the real world and detailed data resource design within the common data architecture.

Reporting and data analysis applications that work closely to tie together company data users and data warehouses need to overcome problem on database performance. Every single day, the amount data collected increases at exponential proportions. Along with the increase, the demands for more detailed reporting and analysis tools also increases.

In a competitive business environment, the areas that are given more focus to gain competitive edge over other companies include the need for timely financial reporting, real time disclosure so that the company can meet compliance regulations and accurate sales and marketing data so the company can grow a larger customer base and thus increase profitability.

Data aggregation helps company data warehouses try to piece together different kinds of data within the data warehouse so that they can have meaning that will be useful as statistical basis for company reporting and analysis. But data aggregation, when not implemented well using good algorithm and tools can lead data reporting inaccuracy.

Ineffective way of data aggregation is one of the major components that can limit performance of database queries.

We can apply the aggregate function for query the result of the database like $\operatorname{sum}(), \operatorname{avg}(), \max ()$ etc. It support the context in clustering so that it effectively done the job.

WSNs is useful for several reasons [Kansal et al. [8] Anastasi et al. [9] ], as discussed below.

Connectivity: As nodes are mobile, a dense WSN architecture may be not a requirement. In fact, mobile elements can cope with isolated regions, so that the constraints on network connectivity can be relaxed, also in terms of nodes (re)deployment. Hence, a sparse WSN architecture becomes a feasible option.

Cost: Since fewer nodes can be deployed, the network cost is reduced in a mobile WSN. Although adding mobility features to the nodes might be expensive, in many cases it is possible to exploit mobile elements which are already present in the sensing area (e.g., trains, buses, shuttles or cars), and attach sensors to them.

Reliability: Since traditional (static) WSNs are dense and the communication paradigm is often (ad hoc) multi-hop, reliability is compromised by interference and collisions. In addition, the 
message loss increases with the number of hops, which may be rather high. Mobile elements, instead, can visit nodes in the network and collect data directly through single-hop transmissions. This reduces not only contention and collisions, but also the message loss.

Energy efficiency: The traffic pattern inherent to WSNs is converge cast, i.e., messages are generated from sensor nodes and are collected by the sink. As a consequence, nodes closer to the sink are more overloaded than others, and subject to premature energy depletion. Since the neighbors of the sink represent the bottleneck of traffic. Mobile elements can help reduce the funneling effect, as they can visit different regions in the network and spread the energy consumption more uniformly, even in the case of a dense WSN architecture.

However, mobility in WSNs also introduces significant challenges which do not arise in static WSNs. These challenges are described below.

Contact detection: Since communication is possible only when the nodes are in the transmission range of each other, it is necessary to detect the presence of a mobile node correctly and efficiently. This is especially true when the duration of contacts is short.

Mobility-aware power management: In some cases, it is possible to exploit the knowledge on the mobility pattern to further optimize the detection of mobile elements. In fact, if visiting times are known or can be predicted with certain accuracy, sensor nodes can be awake only when they expect the mobile element to be in their transmission range.

Reliable data transfer. As available contacts might be scarce and short, there is a need to maximize the number of messages correctly transferred to the sink. In addition, since nodes move during data transfer; message exchange must be mobility-aware.

Mobility control: When the motion of mobile elements can be controlled, a policy for visiting nodes in the network has to be defined. To this end, the path and the speed or sojourn time of mobile nodes have to be defined in order to improve (maximize) the network performance.

\section{LITERATURE SURVEY}

In 2010, YoungSang Yun et al. [10] proposed a framework to maximize the lifetime of the wireless sensor networks (WSNs) by using a mobile sink when the underlying applications tolerate delayed information delivery to the sink. Within a prescribed delay tolerance level, each node does not need to send the data immediately as it becomes available. Instead, the node can store the data temporarily and transmit it when the mobile sink is at the most favorable location for achieving the longest WSN lifetime. To find the best solution within the proposed framework, we formulate optimization problems that maximize the lifetime of the WSN subject to the delay bound constraints, node energy constraints, and flow conservation constraints. They conduct extensive computational experiments on the optimization problems and find that the lifetime can be increased significantly as compared to not only the stationary sink model but also more traditional mobile sink models.

In 2010, Saeed Rasouli Heikalabad et al. [11] proposed the new cluster head selection protocol namely HEECH. This protocol selects a best sensor node in terms of energy and distance as a cluster head. They produce the Simulation Results which show that the HEECH increases the network lifetime about $56 \%$ and $9 \%$ compared to the LEACH and HEED, respectively.

In 2010, Babar Nazir et al. [12] proposed and address hotspot problem and Mobile Sink based Routing Protocol (MSRP) for Prolonging Network Lifetime in Clustered Wireless Sensor 
Network. In MSRP, mobile sink moves in the clustered WSN to collect sensed data from the CHs within its vicinity. During data gathering mobile sink also maintains information about the residual energy of the CHs. Mobile sink based on the residual energy of $\mathrm{CHs}$ move to the $\mathrm{CHs}$ having higher energy. Consequently, the hotspot problem is minimized as the immediate neighbor of the sink is high energy node and it changes because of regular sink movement. It results in a balanced use of WSN energy and improves network life time.

\section{MOBILE SINK WITH JAVA ENVIRONMENT}

The main function of the network layer is routing packets from the source machine to the destination machine, often requiring multiple hops. For broadcast networks routing is an issue if source and destination are not on the same network. The routing algorithm is that part of the network layer software responsible for deciding which output line an incoming packet should be transmitted on. With VC's networks one speaks of session routing, because a route remains in force for an entire user session (e.g. a login session or a file transfer). The following properties are desirable in a routing algorithm:

- correctness and simplicity.

- robustness, against software and hardware failures, traffic changes and topology changes for very long periods.

- stability, some algorithms never converge to an equilibrium.

- fairness and optimality, which are conflicting goals.

Intuitively, increasing the sink velocity $\mathrm{v}$ will improve the system effciency, since in unit time interval the mobile sink can meet more sensors and gather more information throughout the sensor field. However, we should carefully choose this parameter as explained follows. On the one hand, the higher mobile sink velocity, the higher the probability for static sensors to meet mobile sinks. On the other hand, when mobile sinks are moving too fast across the effective communication region of static sensors, there may not be a suffcient long session interval for the sensor and sink to successfully exchange one potentially long packet. In other words, with the increase of sink velocity, the "outage probability" of packet transmission will arise. . Therefore, finding a proper value for sink velocity must be a tradeoff between minimizing the sensor-sink meeting latency and minimizing the outage probability.

Figure 2 further shows the data collection latency, defined as the time differences between when the data is sampled and when they are entered into the DC Genome central database. When using three wireless channels concurrently, over 90 percent of sensor data is collected before the 30 second deadline. 
Data collection delay (3 channels)

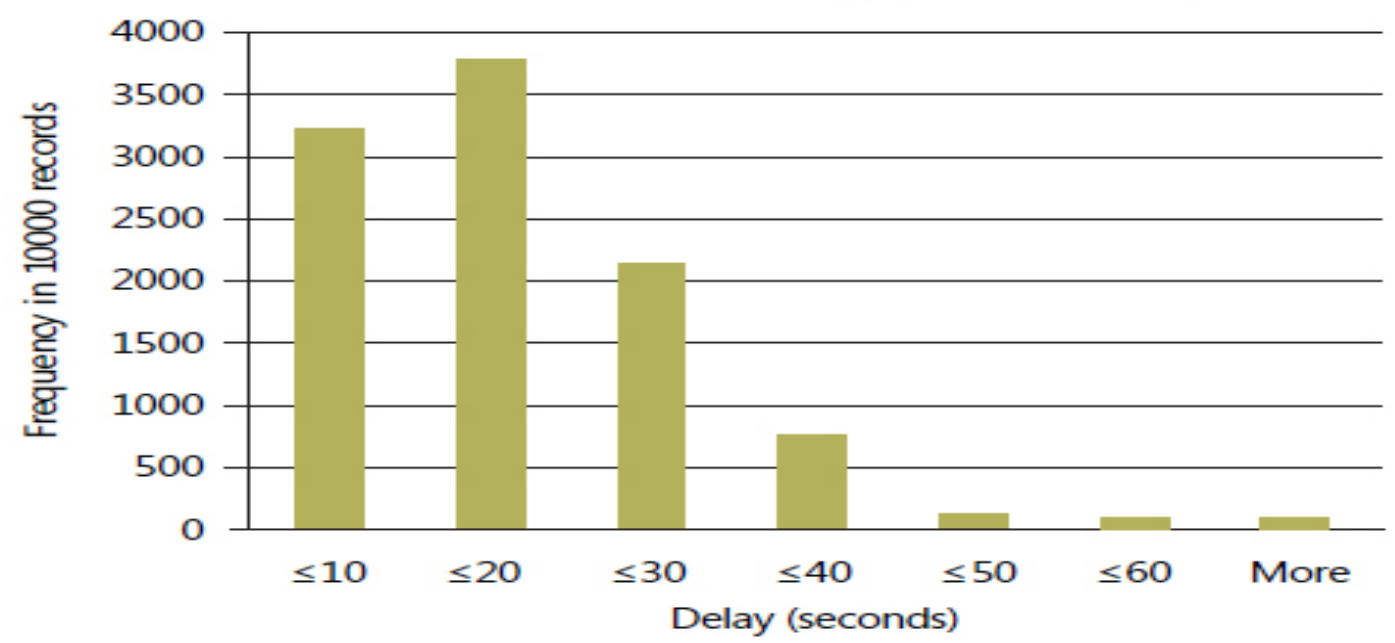

Figure 2: Data collection latency distribution of 10,000 data samples using three wireless channels.

These unprecedented results show that a wireless sensor network can be used to reliably collect environmental data in data centers with low hardware cost and easy installation and maintenance.

Power-efficient gathering for sensor information systems (PEGASIS) is a data-gathering protocol based on the assumption that all sensor nodes know the location of every other node, that is, the topology information is available to all nodes.

Also, any node has the required transmission range to reach the BS in one hop, when it is selected as a leader. The goals of PEGASIS are as follows:

1. Minimize the distance over which each node transmits

2. Minimize the broadcasting overhead

3. Minimize the number of messages that need to be sent to the BS

4. Distribute the energy consumption equally across all nodes

A greedy algorithm is used to construct a chain of sensor nodes, starting from the node farthest from the BS. At each step, the nearest neighbor which has not been visited is added to the chain. The chain is constructed apriori, before data transmission begins, and is reconstructed when nodes die out. 


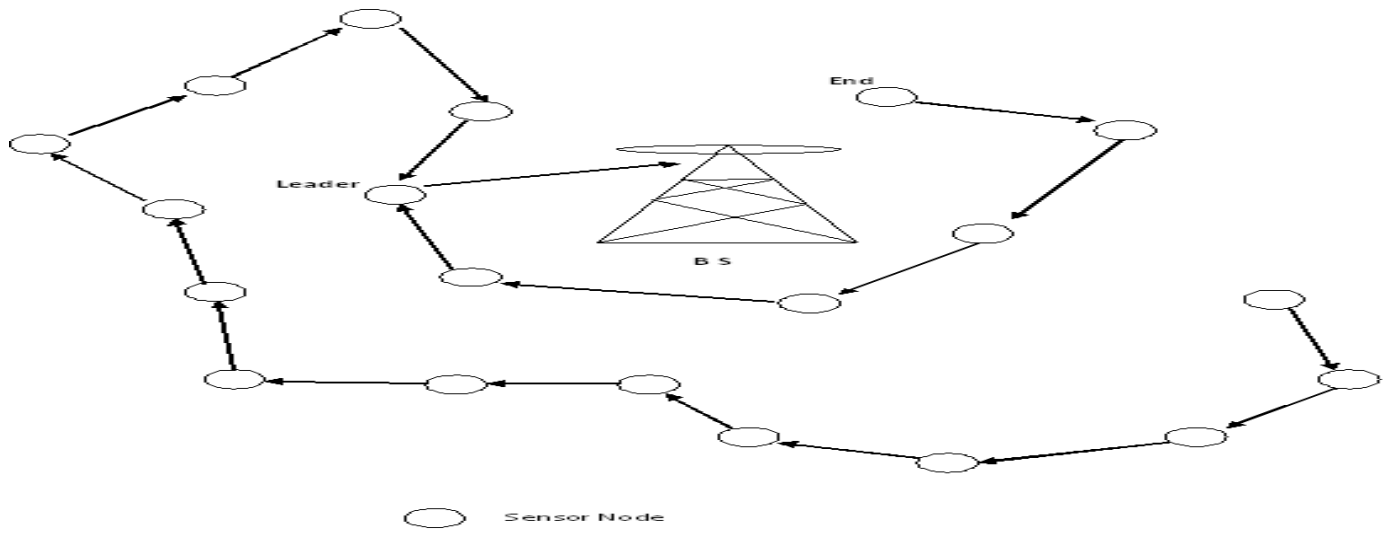

Figure 3: Data gathering with PEGASIS.

At every node, data fusion or aggregation is carried out, so that only one message is passed on from one node to the next. A node which is designated as the leader finally transmits one message to the BS.

Leadership is transferred in sequential order, and a token is passed so that the nodes know in which direction to pass messages in order to reach the leader. A possible chain formation is illustrated in Figure 3 above. The delay involved in messages reaching the $\mathrm{BS}$ is $\mathrm{O}(\mathrm{N})$, where $\mathrm{N}$ is the total number of nodes in the network.

\section{Binary Scheme}

This is also a chain-based scheme like PEGASIS, which classifies nodes into different levels. All nodes which receive messages at one level rise to the next. The number of nodes is halved from one level to the next.

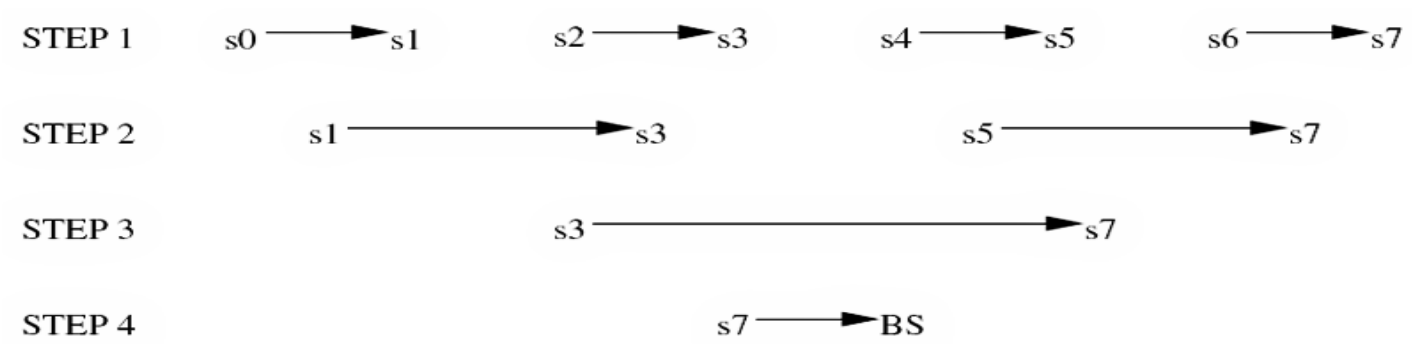

Figure 4: Binary Schemes

For instance, consider a network with eight nodes labeled s0to s7. As Figure 4 above shows, the aggregated data reaches the $\mathrm{BS}$ in four steps, which is $\mathrm{O}(\log 2 \mathrm{~N})$, where $\mathrm{N}$ is the number of nodes in the network. This scheme is possible when nodes communicate using CDMA, so that transmissions of each level can take place simultaneously.

\section{Chain-Based Three-Level Scheme}

For non-CDMA sensor nodes, a binary scheme is not applicable. The chain-based three-level scheme addresses this situation, where again a chain is constructed as in PEGASIS. The chain is 
divided into a number of groups to space out simultaneous transmissions in order to minimize interference. Within a group, nodes transmit one at a time. One node out of each group aggregates data from all group members and rises to the next level.

The index of this leader node is decided apriori. In the second level, all nodes are divided into two groups, and the third level consists of a message exchange between one node from each group of the second level. Finally, the leader transmits a single message to the BS. The working of this scheme is illustrated in Figure 5 below.

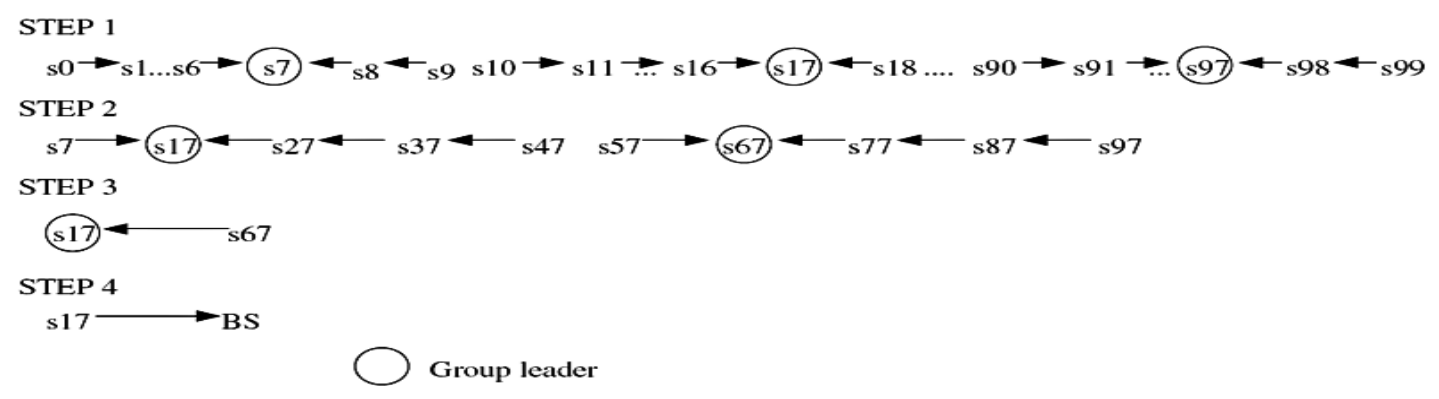

Figure 5: Chain-based three-level schemes

The network has 100 nodes, and the group size is ten for the first level and five for the second level. Three levels have been found to give the optimal energy $\times$ delay through simulations.

In mobile sink environment if we using java based environment then we take the advantage of java security and the flexibility. So it provides also the SQL capabilities in requirement gathering and analysis. There are readymade classes in java which work on TCP/IP protocol which help us to make the connection and work done.

\section{PROPOSED METHOD}

In this section we proposed an Efficient Approach for Data Gathering and Sharing with Inter Node Communication in Mobile-Sink.

Our Proposed algorithm is divided into seven parts:

- Registration Phase

- Authentication Phase

- Request and Reply Phase

- Setup Phase(RS)

- Setup Phase(NN)

- Data Gathering

- Forwarding to Sink

Assumptions

RS-Request sender

NN-New Node

DS- Data Source

UP-User Profile

SP-Service Provider

PR- Probe Request 
PRS-Probe Response

AR-Acknowledge Request

ARS-Acknowledge Response

SSS- Sharing Secret Key

DGPNDS- Data Gathering Protocol with Node Detection and Sharing in Mobile-Sink Based Java Environment

\section{1) Registration Phase}

In this phase, directly user is not eligible. So Admin first register the user with his name, uid, pwd.After the registration user go to the mobile sink java environment. Directly user does not eligible to enter in the DGPNDS environment.

\section{UID-User ID}

\section{PWD-Password}

\section{Computation:}

$1.1 \mathrm{ds}=$ Combination (pwdlluid)

$1.2 \mathrm{up}=\mathrm{ds}[\mathrm{i}$ ] [send to the user profile]

2) Authentication Phase

After the registration phase sender get Uid and Pwd and inter into this phase.

User enters the combination of (pwdlluid)

If(true)

Goto Request and Reply phase

Else

Invalid entry, enter again.

\section{3) Request and Reply Phase}

In this phase we show the request and reply mechanism.

This mechanism is shown in fig 6 .

PR- Probe Request

PRS-Probe Response

AR-Acknowledge Request

ARS-Acknowledge Response

SP-Service Provider

Once the sender becomes active on the medium, it searches for access points or the service provider points in the available management frames known as probe request frames. The probe request frame is sent on every channel the sender supports in an attempt to find all access points. 


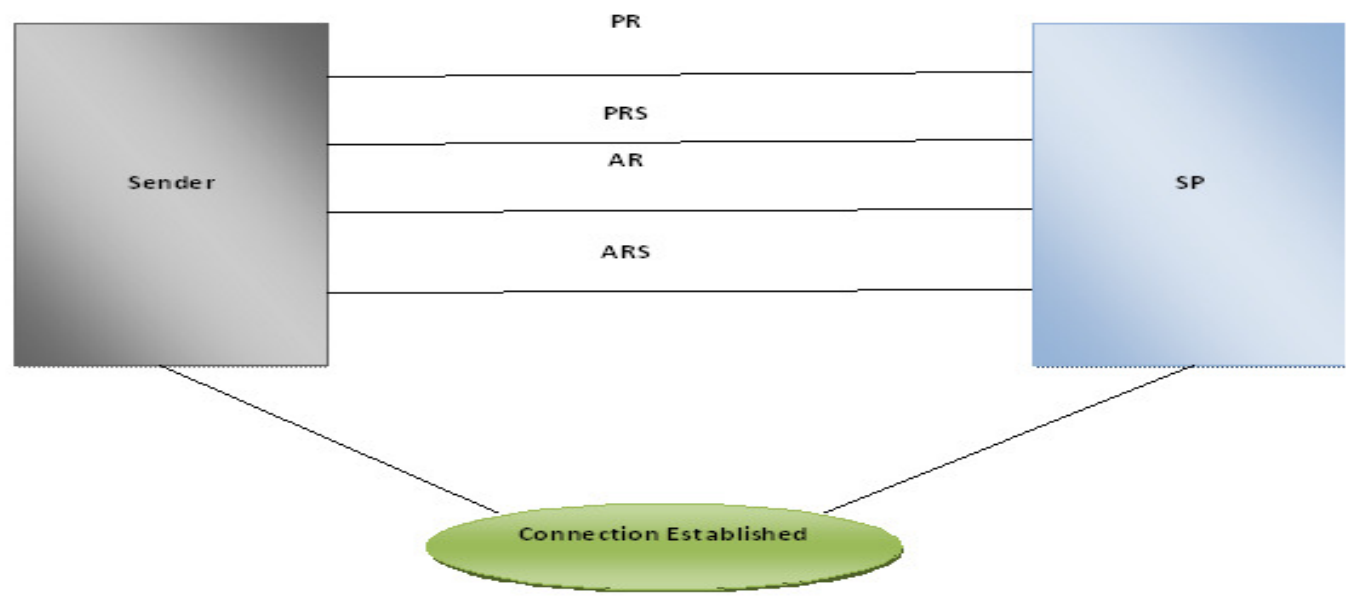

Request and Reply Phase

Figure 6: Requests and Reply Phase

All service provider that are in range (Available list) and match the probe request criteria will respond with a probe response frame containing synchronization information and access point load. The client can determine which access point (SP) to associate to by weighing the supported data rates and access point load.

\section{4) Setup Phase (RS)}

In this phase fig. $3 \mathrm{SP}$ (service provider) wants a shared Secret Key for the next process. Sender sends the Secret Key on the basis of the secret key gathering process is finished. Shared key authentication is the second mode of authentication specified in the 802.11 standard. The Sp request for the shared key otherwise gathering process is not established. The Sender sends the secret key and if it is true then it goes to the next phase.

SSS- Sharing Secret Key

RS-Request sender

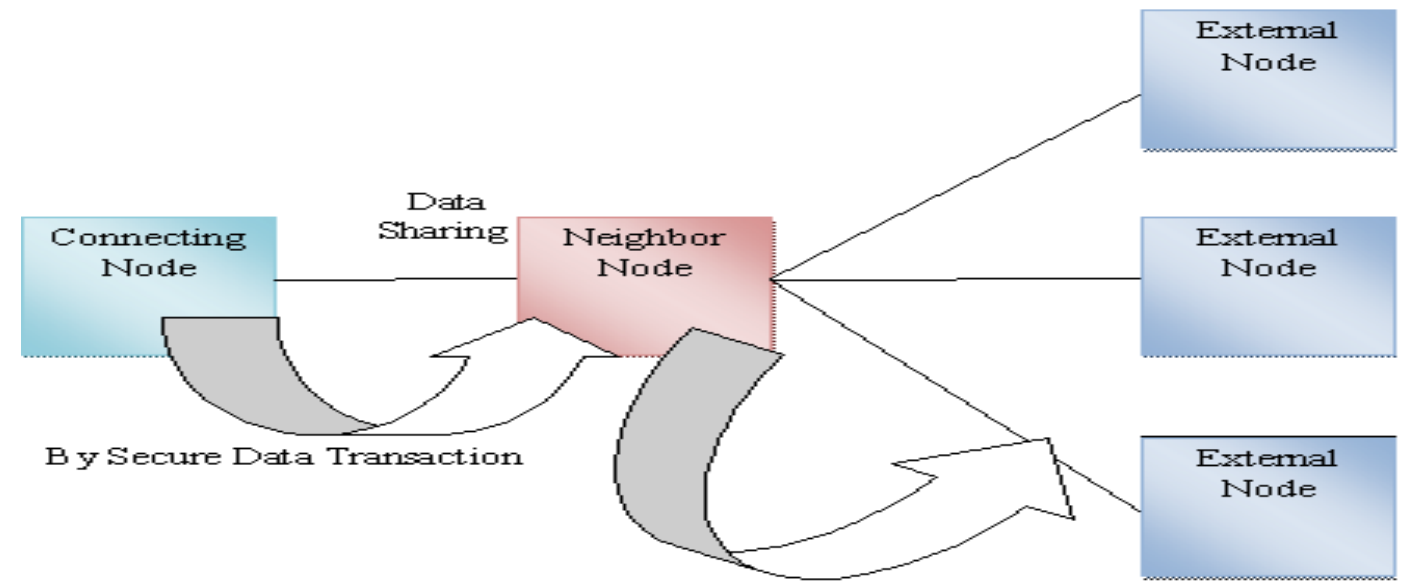

Figure 7: Data Sharing with the Neighbor Node 


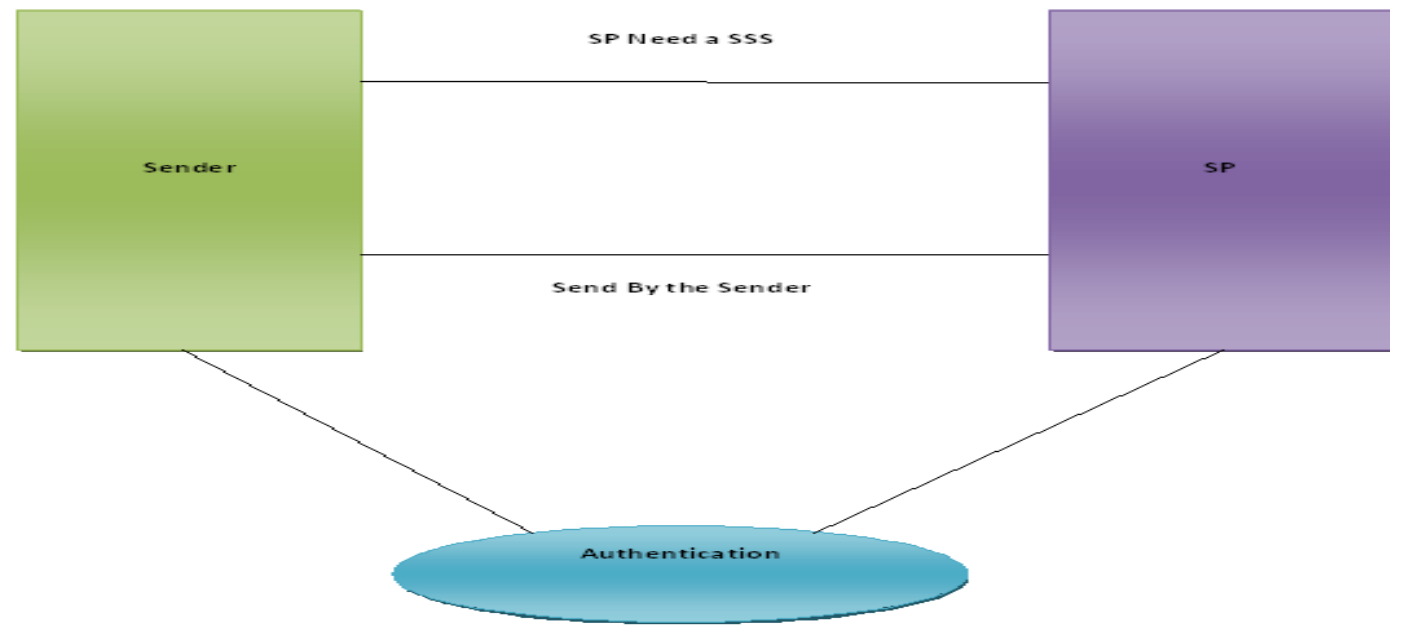

Figure 8: Set up phase

5) Setup Phase(NN)

\section{NN - New Node}

In this phase Service provider attempts to create its new friend list so that it can collect data from different sources so that it becomes available for the sender.

6) Data Gathering

DS- Data Source

In this phase Service provider collects the data which is required by the sender. It aggregates those data for the sender. It then sends the data to the sender, fulfilling his request

\section{7) Forwarding to the Sink}

In this phase next movement decision in first cycle, mobile sink just move to predefined locations in network. Later in second and subsequent cycle node will move to the next access point and in this way scan through the network. It also maintains the record of the communication.

\section{CONCLUSION AND FUTURE ASPECT}

In this paper we proposed an efficient way of data sharing technique, where we gather the data from the neighbour node and by the help of the neighbour node; we can securely access data from the external nodes. So the burden in the neighbour node, which can gather the data for the connecting node. The above phenomena are very secure, because the data gathering is only possible if you have a secure key to transfer the data to the friend node. 


\section{REFERENCES}

[1] I. F. Akyildiz, W. Su, Y. Sankarasubramaniam, and E. Cayirci. A survey on sensor networks. IEEE Communications Magazine, 40:102-114, August 2002.

[2] I. Demirkol, C. Ersoy, and F. AlagOz. Mac protocols for wireless sensor networks: a survey. IEEE Communications Magazine, 44:115-121, April 2006.

[3] K. Akkaya and M. Younis. A survey on routing protocols for wireless sensor networks. Ad Hoc Network Journal, 3:325-349, 2005.

[4] C. Intanagonwiwat, R. Govindan, and D. Estrin. Directed diffusion: A scalable and robust communication paradigm for sensor networks. In Proceedings of Mobicom, pages 56-67, Boston MA, USA, 2000.

[5] W. B. Heinzelman, A. P. Chandrakasan, and H. Balakrishnan. An application-specific protocol architecture for wireless microsensor networks. IEEE Transactions On Wireless Communications, 1:660-670, October 2002.

[6] Communications of the ACM 47 (2004), June. Special Section on Wireless Sensor Networks.

[7] E. Falck, P. Flor_een, P. Kaski, J. Kohonen, P. Orponen, IBalanced data gathering in energyconstrained wireless sensor networks." Proc. First International Workshop on Algorithmic Aspects of Wireless Sensor Networks (ALGOSENSORS 2004), 59\{ 70. Springer-Verlag, LNCS 3121, Berlin, 2004.

[8] Kansal, A., Somasundara, A., Jea, D., Srivastava, M., and Estrin, D. 2004. Intelligent uid infrastructure for embedded networks. In Proceedings of the 2nd ACM International Conference on Mobile Systems, Applications, and Services (MobiSys 2004).

[9] Anastasi, G., Conti, M., and Di Francesco, M. 2009. Reliable and energy-e_cient data collection in sparse sensor networks with mobile elements. Performance Evaluation 66, 12 (December), $791\{810$.

[10] YoungSang Yun, and Ye Xia, "Maximizing the Lifetime of Wireless Sensor Networks with Mobile Sink in Delay-Tolerant Applications", IEEE TRANSACTIONS ON MOBILE COMPUTING, VOL. 9, NO. 9, SEPTEMBER 2010.

[11] Saeed Rasouli Heikalabad, Nasrin Firouz, Ahmad Habibizad Navin and Mir Kamal Mirnia ," HEECH: Hybrid Energy Effective Clustering Hierarchical Protocol for Lifetime prolonging in Wireless Sensor Networks", 2010 International Conference on Computational Intelligence and Communication Networks.

[12] Babar Nazir, Halabi Hasbullah," Mobile Sink based Routing Protocol (MSRP) for Prolonging Network Lifetime in Clustered Wireless Sensor Network", 2010 International Conference on Computer Applications and Industrial Electronics (ICCAIE 2010), December 5-7, 2010, Kuala Lumpur, Malaysia. 